\title{
Hospital Hyperglycemia: Status Investigation and Effect of a Real-time Glycemic Alert System
}

\author{
CHENXIANG CAO ${ }^{1}$, Victor Bernet ${ }^{2}$, Zhaoxiang Liu ${ }^{1}$, Caihong $\mathrm{Li}^{1}$, Chongyang $\mathrm{Bi}^{1}$, \\ Zhenhua Sang ${ }^{1}$, haiyi Liu ${ }^{1}$, and Jianzhong Xiao ${ }^{1}$ \\ ${ }^{1}$ Beijing Tsinghua Changgung Hospital \\ ${ }^{2}$ Mayo Clinic Florida
}

May 22, 2020

\begin{abstract}
OBJECTIVE Hospital hyperglycemia is common and associated with potential adverse outcomes. A Hospital-wide Mobile Phone Alert (HMA) system was built to achieve real time glucose monitoring with warnings for glucose excursions. This study investigated the status of glucose control and evaluated the impact of HMA system on inpatient glycemia management. METHODS Inpatients with hyperglycemia hospitalized between 1 January, 2017 and 31 December, 2018 were identified excluding those $<18$ years of age. The HMA system was activated on 1 October, 2017. It sent real time cellphone warning messages to the patient's designated team physician whenever glucose levels $>10 \mathrm{mmol} / \mathrm{L}$ or $<3 \mathrm{mmol} / \mathrm{L}$ were detected. A serum glucose $>7.8 \mathrm{mmol} / \mathrm{L}$ was defined as hospital hyperglycemia $(\mathrm{HH})$, and $>10 \mathrm{mmol} / \mathrm{L}$ was defined as significant $\mathrm{HH}$ (SHH). Glucose excursions before and after the HMA system was instituted were compared. RESULTS The incidence of HH, SHH and hypoglycemia was $26.1 \%, 12.8 \%$ and $2.5 \%$, respectively. With the HMA system, the monthly glucose related consultation rate for all inpatients increased $65.9 \%$. The rate of $\mathrm{HH}$ glucose amount/ total glucose amount improved with the HMA system, being lower than pre HMA system activation for the surgical wards $(15.8 \pm 4.7 \%$ vs $21.1 \pm 6.1 \%, \mathrm{p}<0.05)$. CONCLUSIONS In this study, one third of inpatients were noted to experience hyperglycemia. Real time cellphone warning messages to the patient's designated team physician can improve consultation utilization for blood glucose excursions. The alert system was found to reduce the incidence of hyperglycemia on surgical wards.
\end{abstract}

\section{Hosted file}

HIS_Main manuscript V_2020.5.20.docx available at https://authorea.com/users/325309/articles/ 453281-hospital-hyperglycemia-status-investigation-and-effect-of-a-real-time-glycemicalert-system 


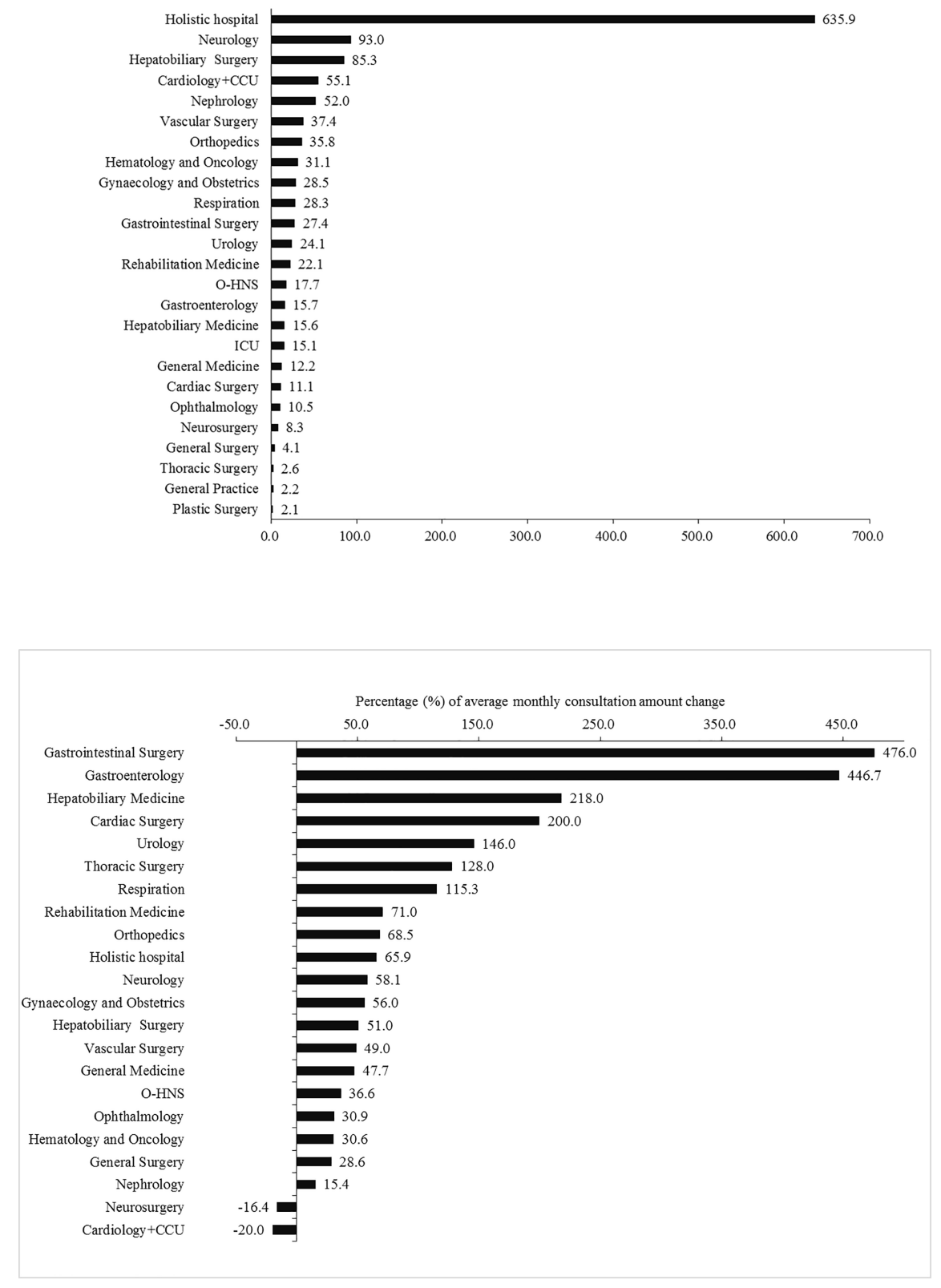

